\title{
ELECTROENCEPHALOGRAM ALTERATIONS DURING PERCEPTION OF PLEASANT AND UNPLEASANT ODORS
}

\author{
Ashkan Yazdani ${ }^{1}$,Eleni Kroupi ${ }^{1}$, Jean-Marc Vesin ${ }^{2}$, Touradj Ebrahimi ${ }^{1}$ \\ ${ }^{1}$ Multimedia Signal Processing Group (MMSPG), Institute of Electrical Engineering, \\ Ecole Polytechnique Fédérale de Lausanne (EPFL), Lausanne, Switzerland. \\ ${ }^{2}$ Applied Signal Processing Group (ASPG), Institute of Electrical Engineering, \\ Ecole Polytechnique Fédérale de Lausanne (EPFL), Lausanne, Switzerland.
}

\begin{abstract}
The olfactory system enables humans and many animals to recognize and categorize different odors and can determine many behavioral and social reactions. For human beings, odor stimuli are highly associated with many processes such as emotions, attraction, mood, etc. One approach to understanding the olfaction is to monitor and analyze human brain activity during perception of odors. In this paper, we analyze the electroencephalogram (EEG) of five subjects during perception of unpleasant and pleasant odor stimuli. We identify the regions of the brain that are active during discrimination of unpleasant and pleasant odor stimuli. We also show that classification of EEG signals during perception of odors can reveal the pleasantness of the odor with relatively high accuracy.
\end{abstract}

Index Terms - Olfaction, Odor, EEG, Brain, Signal Processing

\section{INTRODUCTION}

For many animals the olfactory sense is the most important and thus the most evolved sense. It allows them to interact better with their environment by helping them take right decisions for survival such as to identify food, mates, prays and also escape from their predators. Although human beings appear less driven by olfaction than many other animals, human olfaction has a particular importance and can determine many behavioral and social reactions. Odor stimuli are highly associated with attraction, mood, detection of danger and they have the power to evoke distant memories and boost self-confidence.

Odor stimuli are created by volatilized chemical compounds and are directly perceived by the human olfactory

The research leading to these results has been performed in the frameworks of Swiss National Foundation for Scientific Research (FN 200020132673-1), and the NCCR Interactive Multimodal Information Management (IM2). Furthermore, the authors would like to thank all subjects, who kindly participated in the experiments. Last but not least, we would like thank Dr. Karin Diserens and Dr. Etienne Pralong for their useful inputs on experimental paradigm design. bulb through the olfactory epithelium. In general, odors can be measured from two different aspects, namely odor intensity and odor pleasantness. Odor intensity is perceived as the strength of an odor, and can range from no odor to intolerable. Furthermore, hedonic assessment of odors can scale from extremely unpleasant up to extremely pleasant.

While smelling an odor, olfactory neurons located inside the nasal cavity fire and send special electrical impulses to olfactory bulb. The information then flows from the olfactory bulb to higher cortical areas, such as the piriform cortex [1]. This cortical region has a unique architecture and a unique contribution to odor encoding and perception, so it has been extensively studied [2, 3, 4].

One approach to understanding olfaction and odor perception is to study the changes in the brain electrical activity after smelling an odor. Several studies have tried to capture the oscillatory nature of the olfactory perception by means of EEG signal processing. However, there exists a great deal of variability in terms of the reported findings. For instance, differences in the amount of alpha activity and an increase of theta activity were observed when subjects were exposed to fragrances and the EEG was recorded from 4 electrodes [5]. However, other authors suggested that there are no changes in alpha activity but there are increases in theta activity [5, 6]. Moreover, in a more recent study [7] a decrease in the spectral power of theta and alpha bands was found while smelling essential oils. In the same study, an increase in the high frequency EEG components (11-25 Hz) was reported.

One possible reason for the variability among EEG studies and olfactory psychophysiology is that in addition to the direct activation of the olfactory bulb and the piriform cortex, the human olfactory path is interconnected with other cortical and limbic structures, such as the amygdala [1]. The role of the amygdala in emotion, memory, and autonomic control directly associates olfaction to these processes and adds complexity to the odor perceptual experience. Therefore it is of great importance to investigate the affective dimension of olfactory perception.

In affect recognition research, several different audio/visual 
stimuli are presented to subjects while their brain activity is being recorded. The subjects are asked to provide their selfassessed induced emotions (in terms of pleasantness and excitation), and these data are used to determine the changes in the brain activity during perception of different emotions [8]. The same approach can be used to understand the affective dimension of olfaction. More specifically, similarly to audio/visual stimuli, odors can be classified in terms of their pleasantness. Thus, subjects can be asked to smell different odors and provide their self-assessed perception in terms of pleasantness. These self assessments can be used in order to investigate how odor perception affect brain electrical activity.

Furthermore, most of the previous work in this area, studied the changes in the brain activity after smelling either a pleasant or an unpleasant odor. In other words, EEG changes were studied compared to normal baseline EEG. However, the normal baseline EEG can be modulated by many factors, such as mood, time of the day, tiredness, and many more. Therefore, it is necessary to design an experiment, where all these factors are controlled.

In this work, we propose to study the changes in the brain activity during perception of unpleasant and pleasant odors, in order to deal with the baseline variability problem. More precisely, the EEG changes during stimulation with pleasant odors are compared to the changes during stimulation with unpleasant odors. We aim at exploring the EEG changes in the power spectrum of different brain frequency bands, as well as identifying the brain regions of interest resulting from the difference between perception of pleasant and unpleasant odors. Moreover, in order to explore the possibility of determining the pleasantness of an odor implicitly, only by means of EEG signal processing, a classification problem is posed and the results are presented.

This paper is organized as follows. Section 2 describes the experimental protocol and the signal processing methods used in this study. The results and the further discussion are detailed in Section 3. Finally, the conclusions are presented in Section 4.

\section{MATERIALS AND METHODS}

\subsection{Participants}

Five right-handed male $\mathrm{PhD}$ students and Postdoctoral researchers participated in the study. They were between 26 and 32 years old and were recruited from Swiss Federal Institute of Technology (EPFL). According to their self-reports, none of them had a history of injury in the olfactory bulb or incapability of smelling. All of them stated that they were not suffering from any respiratory, mental or chronic disease. Participants were informed about the protocol and about the purpose of the study, but they were not informed about the odors they would smell. Before the beginning of the experi-

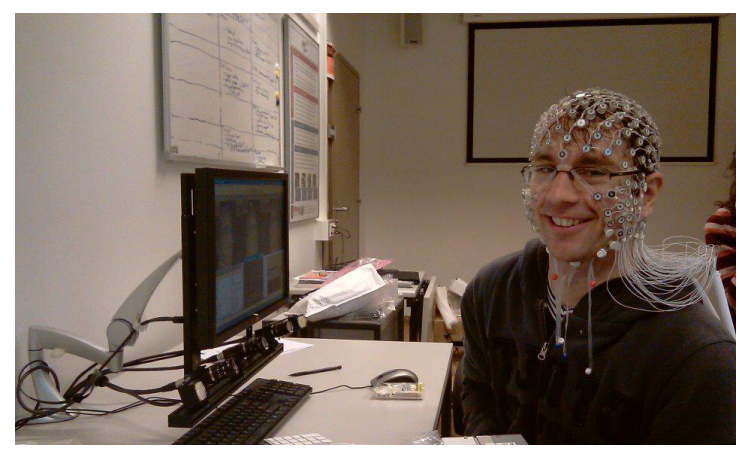

Fig. 1. A participant before the beginning of the experiment.

ments, participants filled in a consent form. During the experiments, participants were seated in a comfortable chair with controlled environment. Finally, neither participants nor experimenters were wearing perfumed products on the day of the experiment.

\subsection{Set up}

The EEG was recorded at $250 \mathrm{~Hz}$ sampling rate from 256 electrodes placed at the standard positions on the scalp. An EGI's Geodesic EEG System (GES) 300 was used to record, amplify, and digitalize the EEG signals. Figure 1 illustrates the EEG set-up and a subject short before the beginning of the experiment.

\subsection{Experimental protocol}

Subjects were asked to close their eyes and breath normally while the experimenter was moving bottles with different odorants towards them to smell. The odorants consisted of valerian, lotus flower, rosewater and fermented Swiss Tomme goat cheese. The odorants were placed inside covered bottles so as to avoid effects of their visual characteristics, such as color and shape.

The experiment consisted of four runs. During each run, after a "smell" command, the experimenter was randomly choosing a bottle with an odor to place it under the subject's nose (1-2 cm under both nostrils) and keep it there for about 2 seconds, constituting a single trial. This process was repeated for twenty to thirty times with the same odor resulting in twenty to thirty single trials.

The time between two single trials of the same odor was set to four seconds in order to avoid adaptation and subject's fatigue. After one run was performed, the subjects were given two minutes break in order to forget the odor (to avoid masking effect) and in order for the odor to be evacuated. During this break, the subjects were asked to rate the odor in terms of intensity and pleasantness, on a scale from 0-10, ranging from no odor to intolerable for intensity, and from extremely unpleasant to extremely pleasant for pleasantness. After this 
break another odor was randomly selected and the same procedure was repeated until all four odors were presented. In this study, only the most pleasant and the most unpleasant odor for each participant were used for data analysis.

\subsection{Signal preprocessing}

Before any processing on the acquired data, the electrodes corresponding to muscle and eye activity were rejected, leading to a total 216 electrodes further analysis. The preprocessing of EEG data was performed in the following order:

\section{High-pass filtering}

In order to remove the slow drifts from the acquired data, it was filtered using a third order Butterworth high-pass filter with cutoff frequency of $3 \mathrm{~Hz}$.

\section{Referencing}

The common average signal of all electrodes was used for referencing.

\section{Single trial extraction}

Single trials of duration $1000 \mathrm{~ms}$ were extracted from the data. Single trials started at stimulus onset. Furthermore, in order to remove the influence of the stimulus-unrelated variations, a one-second baseline was recorded before each run and used later for baselining.

\subsection{Feature extraction and classification}

The power spectral density of the signals and the baseline segments were extracted for frequencies between 4 and 47 $\mathrm{Hz}$, using Welch's non-parametric method with windows of 124 samples and $80 \%$ overlapping. In order to perform the baselining for each run and extract the power changes without considering the pre-stimulus period, the power spectral density of the baseline (1 second before the run) was subtracted from the power spectral density of all single trials in the run. These power changes were captured for different brain frequency bands, namely theta band (4-7 Hz), alpha band (8-13 $\mathrm{Hz}$ ), beta band (14-29 Hz) and gamma band (30-47 Hz). Furthermore, in order to capture changes in the brain activity, associated with processes like workload, engagement and attention, a feature called workload index (WI) [9] was estimated per electrode as follows:

$$
\mathrm{WI}=\frac{\text { beta power }}{\text { theta power }+ \text { alpha power }}
$$

A Support Vector Machine (SVM) classifier with radial basis function (RBF) kernel was used to classify the EEG signals corresponding to pleasant and unpleasant odor perception. Given a training set of instance-label pairs $\left(\mathbf{x}_{i}, y_{i}\right), i=$ $1, \ldots, l$ where $x_{i} \in \mathbb{R}^{n}$ and $y \in\{1,-1\}^{l}$, denote the n-dimensio -nal feature vector and ground-truth, respectively, and $l$ is the number of trials, SVMs acquire the solution of the following optimization problem $[10,11]$ :

$$
\begin{array}{ll}
\min _{\mathbf{w}, b, \boldsymbol{\xi}} & \frac{1}{2} \mathbf{w}^{T} \mathbf{w}+C \sum_{i=1}^{l} \xi_{i} \ldots \\
\text { subject to } & y_{i}\left(\mathbf{w}^{T} \phi\left(x_{i}\right)+b\right) \geq 1-\xi_{i}, \ldots \\
& \xi_{i} \geq 0,
\end{array}
$$

where $\mathbf{w}$ is a vector that represents the adjustable parameters, $\phi\left(x_{i}\right)$ represents the predefined functions of $\mathbf{x}, b$ is a bias and $C$ is the penalty parameter. RBF kernel (with penalty parameter $C$ and kernel parameter $\gamma$ ) was used due to the fact that the number of features was not very large and the fact that RBF kernel considers possible non-linear relationship between class labels and features. LIBSVM package was used for this study [12].

\section{RESULTS AND DISCUSSION}

The Hilbert-Schmidt independence criterion (HSIC) [13] was used in order to investigate the dependence between EEG segments corresponding to the pleasant and the unpleasant odor stimuli across subjects, for each brain frequency band. The HSIC test was selected because it outperforms traditional correlation tests and because it considers the subjects as i.i.d samples. The HSIC test uses a kernel independence measure in order to detect linear or non-linear dependencies between arbitrary input variables. Furthermore, the HSIC test either rejects or accepts the null hypothesis depending on a certain threshold. Following [13], the null hypothesis was that the pleasant and the unpleasant odors are independent. The $p$-value of the HSIC test was computed using Bootstrap approximation with $10^{4}$ re-sampling repetitions and the confidence interval was set to $95 \%$ to avoid Type II errors, where a false hypothesis might not be rejected. Finally, the kernel size of the HSIC test was set to the median distance between the points [13].

HSIC test revealed significant independence between the pleasant and unpleasant odor perception across subjects for all brain frequency bands. Figures 2-6 illustrate the separability of brain activity when stimulated with pleasant and unpleasant odors. More precisely, each color represents the degree of separability of the extracted EEG features during perception of hedonically different odors. For the measure of separability, the fisher discriminant ratio (FDR) [14] of each electrode $i$ was computed as follows:

$$
\mathrm{FDR}_{i}=\frac{\left(m_{p, i}-m_{u, i}\right)^{2}}{\sigma_{p, i}^{2}+\sigma_{u, i}^{2}}
$$

where $m_{p, i}$ and $\sigma_{p, i}$ represent the mean and the variance values of EEG single trials powers acquired from electrode $i$ during stimulation with pleasant odors and $m_{u, i}$ and $\sigma_{u, i}$ represent the mean and the variance values of EEG single trials 


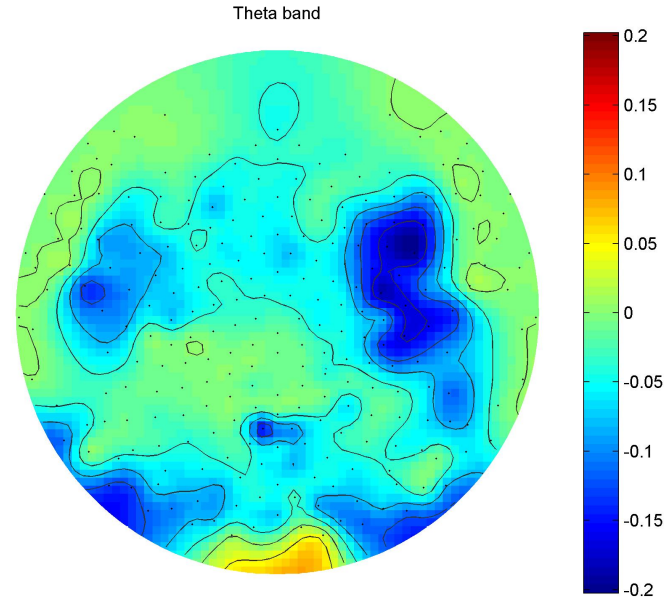

Fig. 2. Separability of theta band EEG during perception of unpleasant and pleasant odors

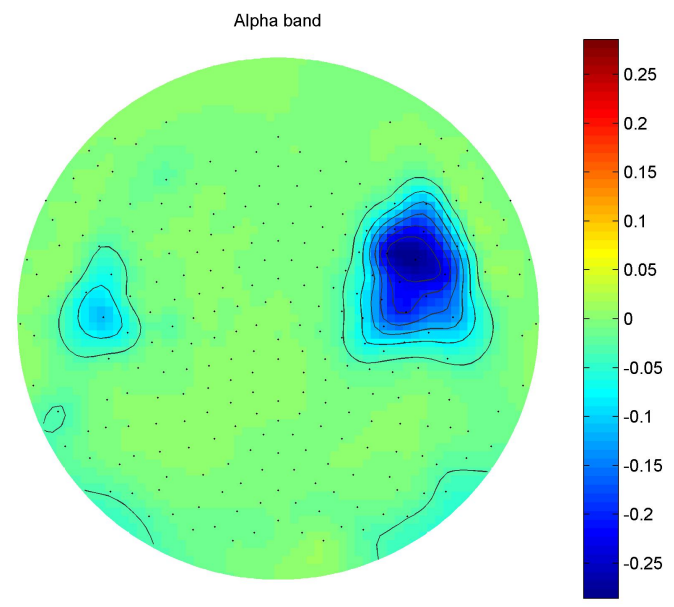

Fig. 3. Separability of alpha band EEG during during perception of unpleasant and pleasant odors

powers acquired from the same electrode when stimulated with unpleasant odors. The larger the value of $\mathrm{FDR}_{i}$, the more separable the EEG signals during pleasant and unpleasant odor perception. Intuitively, as can be seen from the mathematical description of the metric, the two classes are more separable when their mean values are far from each other and their variances are as small as possible. In Figures 2-6, the signed values of FDR, denoted by SFDR, are represented by colors. The signed values of FDR is defined as follows:

$$
\operatorname{SFDR}_{i}=\operatorname{sign}\left(m_{p, i}-m_{u, i}\right) \times \mathrm{FDR}_{i}
$$

where $\operatorname{sign}(x)$ equals +1 and -1 for positive and negative values of $x$, respectively. Small values of SFDR implies the insignificant separability of the EEG signals during pleasant and unpleasant odor perception, whereas large values of SFDR suggest a good separability between these two classes. The

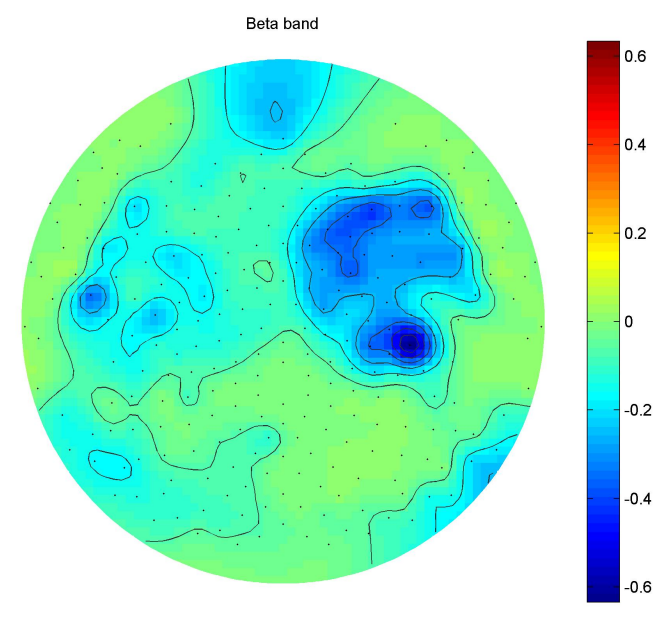

Fig. 4. Separability of beta band EEG during perception of unpleasant and pleasant odors

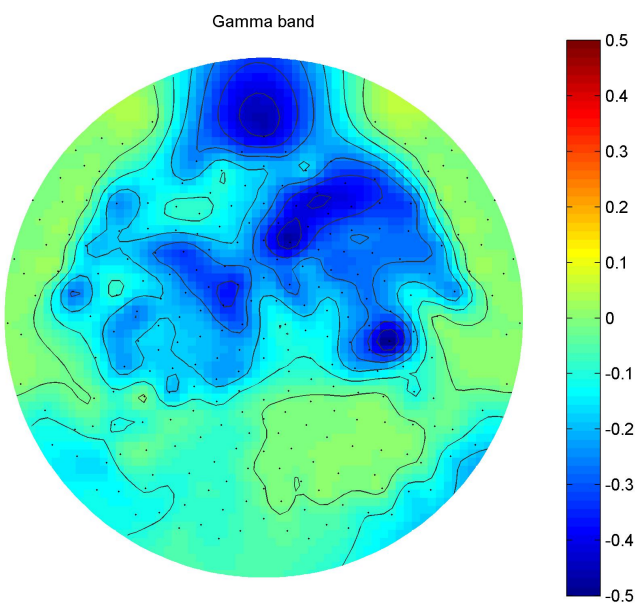

Fig. 5. Separability of gamma band EEG during perception of unpleasant and pleasant odors

positive and negative signs of SFDR values demonstrate the increase and decrease in the brain activation respectively, during pleasant versus unpleasant odor perception.

As it can be seen in these figures, when smelling an unpleasant odor, the brain activity increases in frontal and central regions for almost all bands. This increase in the EEG power is also an unilateral increase, creating an asymmetry in the brain activity distribution. As the increase in the brain power occurs in the right hemisphere, our results are consistent with the results of the research on emotions and affective computing, demonstrating a negative affect or withdrawal situation created after smelling an unpleasant odor $[15,16,17$, $18]$.

In order to perform single trial classification, the leaveone-subject-out cross validation scheme was considered. In other words, a classifier was trained based on the EEG sig- 


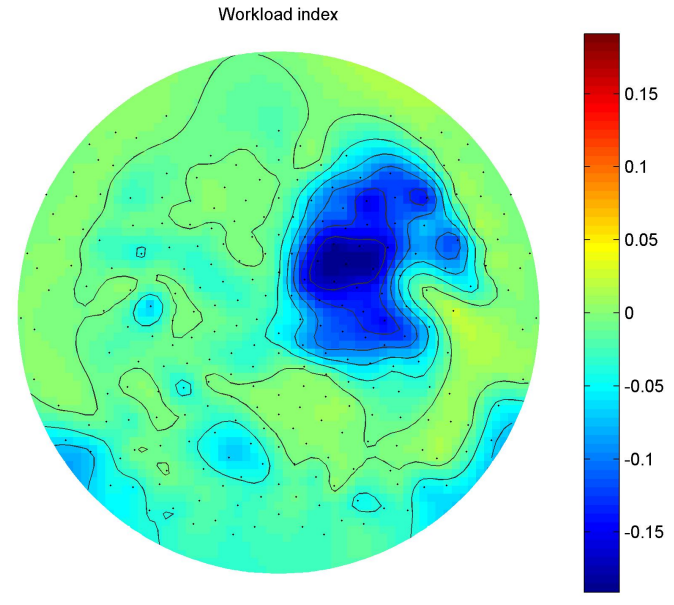

Fig. 6. Separability of workload index during perception of unpleasant and pleasant odors

Table 1. Single trial classification results of EEG signals using features of different frequency bands

\begin{tabular}{|c|c|c|c|c|c||c|}
\hline & Subject1 & Subject2 & Subject3 & Subject4 & Subject5 & Average \\
\hline Theta & $70.00 \%$ & $58.00 \%$ & $50.00 \%$ & $68.57 \%$ & $50.00 \%$ & $59.31 \%$ \\
\hline Alpha & $77.50 \%$ & $66.00 \%$ & $57.50 \%$ & $62.86 \%$ & $50.00 \%$ & $62.77 \%$ \\
\hline Beta & $67.50 \%$ & $58.00 \%$ & $87.50 \%$ & $51.43 \%$ & $100 \%$ & $72.89 \%$ \\
\hline Gamma & $62.50 \%$ & $58.00 \%$ & $55.00 \%$ & $60.00 \%$ & $100 \%$ & $67.10 \%$ \\
\hline W.I & $55.00 \%$ & $64.00 \%$ & $62.50 \%$ & $54.29 \%$ & $75.00 \%$ & $62.16 \%$ \\
\hline
\end{tabular}

nals of four subjects and the EEG signal of the remaining subject was used as the test set. This was repeated until all subjects had been considered as the test set once. The leave-one-subject-out cross validation scheme provides information about subject-independent EEG changes and explores the possibility of developing a generic classifier, which can automatically classify EEG signals of a new naive subject without any other training. The single trial classification results are presented in Table 1.

As it can be seen in this table, the results of single trial classification suggest that using the information in the beta and gamma bands result in relatively high classification accuracies. It can also be observed that the classification results vary among the subjects, suggesting that some subjects have very different EEG benchmarks during perception of pleasant and unpleasant odor stimuli. One approach to deal with this problem, is to repeat the methodology introduced in this paper with a larger number of subjects. In this way, it could be guaranteed that there are clusters of subjects with similar EEG benchmarks and that the training set is comprehensive enough to include all profiles.

\section{CONCLUSION}

In this paper, we explored the EEG alterations during perception of pleasant and unpleasant odors. To this end, a sub- jective test was designed and five subjects participated in a test, where they were asked to smell pleasant and unpleasant odors while their EEG signal was being recorded. Analysis of the recorded data demonstrated that there is a unilateral right frontal activation in the brain activity during perception of unpleasant odors. This is of significant interest, as it is in consistency with the outcomes of emotion recognition studies, where it is shown that frontal activation on the right hemisphere is created by a withdrawal-related emotion. The results of this study suggest the feasibility of EEG signal processing as a tool to inquire about the pleasantness of an odor for a given subject. Further extension of this work can be to use the proposed methodology for assessment of various odors and to explore whether it is possible to extract information about odor pleasantness on a continuous scale. If the answer to the latter is positive, the proposed methodology in this paper can be used for implicit quality assessment of different odors in terms of their pleasantness.

\section{REFERENCES}

[1] D.A. Wilson and R.J. Stevenson, "Learning to smell," Baltimore: Johns Hopkins UP, 2006.

[2] C. Zelano, J. Montag, B. Johnson, R. Khan, and N. Sobel, "Dissociated representations of irritation and valence in human primary olfactory cortex," Journal of neurophysiology, vol. 97, no. 3, pp. 1969-1976, 2007.

[3] J.D. Howard, J. Plailly, M. Grueschow, J.D. Haynes, and J.A. Gottfried, "Odor quality coding and categorization in human posterior piriform cortex," Nature neuroscience, vol. 12, no. 7, pp. 932-938, 2009.

[4] M. Bensafi, "The role of the piriform cortex in human olfactory perception: Insights from functional neuroimaging studies," Chemosensory Perception, pp. 1-7, 2011.

[5] T.S. Lorig and G.E. Schwartz, "EEG activity during relaxation and food imagery.," Imagination, Cognition and Personality, 1988.

[6] WR Klemm, SD Lutes, DV Hendrix, and S. Warrenburg, "Topographical EEG maps of human responses to odors," Chemical senses, vol. 17, no. 3, pp. 347, 1992.

[7] AA Cherninskii, IG Zima, N.Y. Makarchouk, NG Piskorskaya, and SA Kryzhanovskii, "Modifications of EEG related to directed perception and analysis of olfactory information in humans," Neurophysiology, vol. 41, no. 1, pp. 63-70, 2009.

[8] S. Koelstra, C. Muhl, M. Soleymani, J. Lee, A. Yazdani, T. Ebrahimi, T. Pun, A. Nijholt, and I. Patras, "Deap: A 
database for emotion analysis using physiological signals," Affective Computing, IEEE Transactions on, , no. 99, pp. 1-8, 2011.

[9] C. Berka, D.J. Levendowski, M.M. Cvetinovic, M.M. Petrovic, G. Davis, M.N. Lumicao, V.T. Zivkovic, M.V. Popovic, and R. Olmstead, "Real-time analysis of EEG indexes of alertness, cognition, and memory acquired with a wireless EEG headset," International Journal of Human-Computer Interaction, vol. 17, no. 2, pp. 151170, 2004.

[10] B.E. Boser, I.M. Guyon, and V.N. Vapnik, "A training algorithm for optimal margin classifiers," in Proceedings of the fifth annual workshop on Computational learning theory. ACM, 1992, pp. 144-152.

[11] C. Cortes and V. Vapnik, "Support-vector networks," Machine learning, vol. 20, no. 3, pp. 273-297, 1995.

[12] C.C. Chang and C.J. Lin, "LIBSVM: a library for support vector machines," Software available at http: //www.csie.ntu.edu.tw/ cjlin/libsvm/, 2001.

[13] A. Gretton, K. Fukumizu, C.H. Teo, L. Song, B. Schölkopf, and A.J. Smola, "A kernel statistical test of independence," 2008.

[14] S. Theodoridis and K. Koutroumbas, "Pattern recognition (fourth)," 1998.

[15] N.H. Kalin, C. Larson, S.E. Shelton, and R.J. Davidson, "Asymmetric frontal brain activity, cortisol, and behavior associated with fearful temperament in rhesus monkeys.," Behavioral Neuroscience, vol. 112, no. 2, pp. 286, 1998.

[16] R.E. Wheeler, R.J. Davidson, and A.J. Tomarken, "Frontal brain asymmetry and emotional reactivity: A biological substrate of affective style," Psychophysiology, vol. 30, no. 1, pp. 82-89, 1993.

[17] J.A. Coan and J.J.B. Allen, "Frontal eeg asymmetry as a moderator and mediator of emotion," Biological psychology, vol. 67, no. 1-2, pp. 7-50, 2004.

[18] R.J. Davidson, P. Ekman, C.D. Saron, J.A. Senulis, and W.V. Friesen, "Approach-withdrawal and cerebral asymmetry: Emotional expression and brain physiology: I.," Journal of personality and social psychology, vol. 58, no. 2, pp. 330, 1990. 